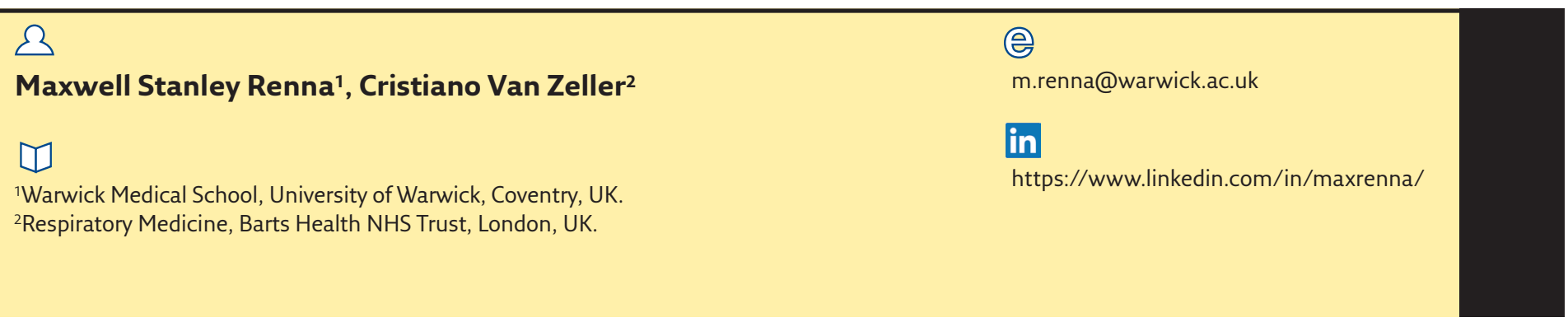

\title{
Does medical school lung function teaching need an update?
}

\section{Introduction to the uses of lung function}

Dyspnoea, defined as breathing difficulty or discomfort [1], is a common symptom of cardiorespiratory distress, seen in $\sim 27 \%$ of the global population [2]. Furthermore, lung disease is becoming one of the most prevalent types of disease globally, with chronic obstructive pulmonary disease (COPD) expected to become the third commonest cause of death by 2030 [3]. This underlines the importance of ensuring accurate diagnostic testing of lung function. Investigations such as pulse oximetry, chest radiography, ECGs and spirometry are some of the recommended tests to investigate the cause of suspected dyspnoea [4]. When COPD is suspected, alongside patient history of exposure to harmful chemicals and lifestyle choices, one of the most crucial diagnostic tools for measuring lung function is the use of spirometry for dynamic lung volume measurements. Broadly, pulmonary function tests (PFTs) categorise lung pathologies into obstructive or restrictive patterns based on the forced expiratory volume in $1 \mathrm{~s}(\mathrm{FEV} 1)$ or forced vital capacity (FVC) or the ratio between FEV1/FVC. Examples of common obstructive pathologies include asthma, COPD and bronchiectasis; common restrictive diseases include pulmonary fibrosis, asbestosis and sarcoidosis [1]
The uses of lung function testing, in general, include the detection of the presence or absence of disease, assessment of disease progression, and responses to treatment as part of preoperative assessments for routine patients and those who may be at elevated risk of anaesthesia complications. It is important that medical students are taught up to date diagnostic criteria as building blocks for further clinical training.

\section{Current taught diagnostic criteria}

The current taught criteria for medical students focuses on using predicted values to categorise obstructive diseases based on the FEV1/FVC ratio or the FEV 1 value alone. They state that a normal lung has a $\mathrm{FEV}_{1}>80 \%$ of the predicted values, a FVC $>80 \%$ or a FEV1/FVC $>0.7$. Conversely, for restrictive patterns, a FEV1/FVC $>0.7(70 \%)$ with a reduced FVC would suggest the presence of disease. This information is based on the 1983 European Community for Coal and Steel (ECCS) reference values and the 1993 European Respiratory Society (ERS) update. This is the more traditional method of assessing lung function and the criteria offer a basic introduction to lung function testing, but basing clinical knowledge on outdated criteria
Cite as: Renna MS,

Van Zeller C. Does medical school lung function teaching need an update? Breathe 2018; 14: e1-e3. 
poses problems when it comes to clinical practice and development of services within an already constrained National Health Service (NHS) environment.

There are also a few issues with using these diagnostic criteria, the first being that the predicted values do not take into consideration ethnic variations without manual inputting of values, which can sometimes be erroneously done. Another issue is that the predicted values are not accurate for patients under the age of 18 or over the age of 70 years. A further issue pertains to the accuracy of using percentage predicted values as diagnostic criteria over other statistical methods, with traditional methods leading to higher rates of false positive diagnosis [5]. With an increasing number of patients over the age of 70 years expected to be performing lung function tests over the next decade, the introduction of more reliable ranges is important for expediting their use in clinical practice and for standardisation of testing.

\section{The solution?}

More recently, more accurate methods of assessing lung function have been developed by the Global Lung Initiative (GLI) with their work on incorporating statistics into diagnostics and diagnosing patients based on their standard residual value, also known as z-scores, which considers lower limits of normal and assesses how far away from the normal distribution the value sits [6]. This means that diagnosing lung conditions can be carried out with more accuracy and take into consideration a more accurate normal variation within the whole population.

The GLI comprises an updated set of predicted values, currently available for spirometry and diffusion capacity testing, which includes multiethnic equations for both males and females

Table 1 Comparison of GOLD spirometry criteria versus the standard residual (z-score) ranges

\begin{tabular}{lccc}
\hline Severity & $\begin{array}{c}\text { Percentage } \\
\text { predicted }\end{array}$ & Severity & $\begin{array}{c}\text { Standard residual/ } \\
\text { z-score }\end{array}$ \\
\hline Normal & $\mathrm{FEV} 1 / \mathrm{FVC}>0.7$ & Normal & \pm 1.645 \\
& $\mathrm{FEV} 1>80 \%$ & & \\
Mild COPD & $\mathrm{FEV} 1 / \mathrm{FV} C<0.7$ & Mild & $\pm 1.645-2.500$ \\
& $\mathrm{FEV} 180 \%$ & & \\
Moderate COPD & $\mathrm{FEV} 1 / \mathrm{FV} C<0.7$ & Moderate & $\pm 2.510-3.500$ \\
& $50 \%<\mathrm{FEV} 1<80 \%$ & & \\
Severe COPD & $\mathrm{FEV} 1 / \mathrm{FV}<<0.7$ & Severe & $\pm>3.500$ \\
& $30 \%<\mathrm{FEV} 1<50 \%$ & & \\
Very severe COPD & $\mathrm{FEV} 1 / \mathrm{FV} C<0.7$ & & \\
& $\mathrm{FEV} 1<30 \%$ & &
\end{tabular}

between the ages of 3 and 95 years [6]. Hence, there is a more accurate and less error prone method of generating predicted values. With the majority of respiratory function machines having the $\mathrm{GLI}$ ranges embedded in their software and these predicted ranges being endorsed by the American Thoracic Society (ATS), Australian and New Zealand Society of Respiratory Science (ANZSRS), Asian Pacific Society for Respirology (APSR) and the Thoracic Society of Australia and New Zealand (TSANZ), now appears to be the ideal time to introduce these diagnostic criteria into medical school teaching [6].

Traditionally, although predicted values consider normal variation in the production of their reference ranges, their population was targeted at Caucasian males, not representative of the global population, who present with varying values depending on the demographic. There are also differing percentage cut-offs for different tests, for example spirometry and diffusion capacity. Moreover, the use of standard residuals means a patient's deviation can be measured from a range of reference values, independent of ethnicity bias and offers a more accurate way of measuring whether a patient's result is abnormal or not.

In clinical practice, this means that the traditional GOLD (Global Initiative for Chronic Obstructive Lung Disease) spirometry criteria can be taught, with the standard residual values, as suggested by KINNEAR et al. [7] or by QuANJer et al. [8] with an emphasis on the new endorsed $\mathrm{GLI}$ values that will enter clinical practice (table 1) [7-9]. Not only can this be used for spirometry, having a single standard residual cutoff applied to diffusion capacity and lung volumes means that instead of three different predicted value thresholds, a single diagnostic value can be applied across the majority of lung function tests. This will enable more appropriate diagnosis and accurate measurement of a patient's lung function over time across any parameter and will ultimately simplify diagnosing respiratory conditions and reduce erroneous diagnoses.

\section{What does this mean for clinical practice?}

If the example of a 94-year-old female patient is used, her values are likely to present as inaccurate when using the traditional method in comparison to when using the $\mathrm{GLI}$ predicted values as she exceeds the upper age limit. Moreover, her percentage of predicted values may well rise, even when her lung function decreases due to the inaccuracies of using percentage predicted values over standard residual monitoring. In this instance, medical students should be taught to look at both absolute values when dealing with trends, but also the GLI reference ranges 
(or standard residual) in addition to the current diagnostic criteria.

\section{Conclusion}

In conclusion, teaching what is deemed to be simpler diagnostic criteria early on opens avenues for potential harm in the long run for patients seen in respiratory clinics by junior and foundation doctors. There is an argument to update medical teaching to incorporate new diagnostic criteria to reduce the potential harm posed and, as with all diagnostic criteria, the clinician's judgement is paramount to providing effective clinical practice. As the GLI values have accreditation from many established organisations across the world, there is a perfect opportunity to expedite the implementation of these values while improving the accuracy of diagnosis and provide a universal scoring system for use in respiratory clinics by introducing these diagnostic criteria in early training.

\section{Conflict of interest}

None declared.

\section{References}

1. Spiro SG, Silvestri GA, Agusti A. Clinical Respiratory Medicine. 4th Edn. Philadelphia, Elsevier, 2012.

2. Gonseth R, Vollmer WM, Hardie JA, et al. Predictors of dyspnoea prevalence: results from the BOLD study. Eur Respir J 2014; 43: $1610-1620$

3. WHO. World Health Statistics. Geneva, WHO, 2008.

4. Kuzniar TJ. Assessment of dyspnoea, BMJ Best Practice. London, BMJ Publishing Group, 2017. Available from: http:// bestpractice.bmj.com/topics/en-gb/862

5. Swanney MP, Ruppel G, Enright PL, et al. Using the lower limit of normal for the FEV1/FVC ratio reduces the misclassification of airway obstruction. Thorax 2008; 63: 1046-1051.
6. Quanjer PH, Stanojevic S, Cole TJ, et al. Multi-ethnic reference values for spirometry for the 3-95-yr age range: the global lung function 2012 equations. Eur Respir J 2012; 40: 1324-1343.

7. Kinnear WJM. Lung function tests: A guide to their interpretation. Nottingham, Nottingham University Press, 1997.

8. Quanjer PH, Pretto JJ, Brazzale DJ, et al. Grading the severity of airways obstruction: new wine in new bottles. Eur Respir $J$ 2014; 43: 505-512.

9. GOLD. Global strategy for the diagnosis, management, and prevention of chronic obstructive pulmonary disease. 2008. Available from: http://goldcopd.org 